\title{
Clinical Management of Medical Disorders in Orthodontics
}

\author{
Anila Charles $^{1, *}$, Senkutvan RS $^{1}$, Sanjay Jacob ${ }^{1}$, Krishnan C.S ${ }^{2}$, Sivaram Subbiah ${ }^{3}$ \\ ${ }^{1}$ Department of Orthodontia, Madha Dental College, M.G.R University, Chennai \\ ${ }^{2}$ Department of Periodontics, Madha Dental College, M.G.R University, Chennai \\ ${ }^{3}$ Department of Orthodontics Penang International Dental College \\ *Corresponding author: cs_anila@yahoo.co.in
}

Received January 08, 2014; Revised April 14, 2014; Accepted April 16, 2014

\begin{abstract}
Orthodontists are confronted with an increasing number of medically compromised patients who require proper alignment for esthetics and oral rehabilitation. The present review is regarding the current knowledge of the most common systemic and local diseases on the outcome of orthodontic treatment, Fortunately in orthodontics complications are infrequent and are of minor consequence, nevertheless the practitioner should understand and have a basic knowledge and understanding of the disease and its impact on the oral cavity as it is essential to recognize oral signs and symptoms of undiagnosed medical problems too.
\end{abstract}

Keywords: medical disorders, orthodontic treatment, guidelines

Cite This Article: Anila Charles, Senkutvan RS, Sanjay Jacob, Krishnan C.S, and Sivaram Subbiah, "Clinical Management of Medical Disorders in Orthodontics.” International Journal of Dental Sciences and Research, vol. 2, no. 2 (2014):36-41. doi: 10.12691/ijdsr-2-2-3.

\section{Introduction}

Optimal oral health is essential for all patients and medically compromised require prior investigation before commencement of orthodontic treatment. Since orthodontics deals with aesthetics, it seems reasonable to assume that children and adult in spite of their medical conditions they want to benefit from orthodontic treatment.

Medical conditions commonly encountered in orthodontic patients include Risk of Infective endocarditis / Hypertension/ Diab tes/ Thyroid disease/ Epilepcy/ Pregnancy. As a rule, general medical problem can affect orthodontic treatment and care should be taken while managing medically compromised patients as it is not an absolute contraindication.

\subsection{Infective Endocarditis}

Infective endocarditis (IE) is defined as an infection of the endocardial surface of the heart, which may include one or more heart valves, the mural endocardium, or a septal defect. Its intracardiac effects include severe valvular insufficiency, which may lead to intractable congestive heart failure and myocardial abscesses [1]. Endocarditis can be divided into a sub acute, Acute and Post-operative endocarditis. The causative organisms are usually the streptococci, staphylococci, Rickettsiae, Chlamydia or fungus [2]. Endocarditis commonly affects heart valves, nonvalvular areas or mechanical devices that are implanted in the heart, such as artificial heart valves, pacemakers, or implantable defibrillators [3].
Endocarditis occurs when bacteria enter the bloodstream and attach to a damaged portion of the inner lining of the heart or abnormal heart valves, not all bacteria entering the bloodstream are capable of causing endocarditis. Only those bacteria that are able to stick to the surface lining of the heart and to abnormal valves tend to cause endocarditis. The ability of these bacteria to stick to the surface lining is aided by a pre existing microscopic clot that often forms at this abnormal sites [4].

The transient becteremia usually result on the lining of mouth, intestinal tract, minor cuts on our day to day activities.eg brushing teeth or chewing Although uncommon, endocarditis is important because, despite antimicrobial therapy, it can result in serious complications such as stroke or even death. Patient seeking orthodontic treatment should be made aware of the risk of endocarditis, the need to avoid bacteraemia and the importance of maintaining good oral hygiene as the bacteraemia experienced by the patient may be increased by plaque accumulation which can be greater in the presence of orthodontic appliances. Hence any invasive procedures that may cause transient bacteraemia should be accompanied by appropriate antibiotic prophylaxis [5].

\subsection{Regimens for Dental Procedures}

Amoxicillin 2 g (child $50 \mathrm{mg} / \mathrm{kg}$ ) oral 1 hour preprocedure (i.v 30 mins before) Alternative: Clindamycin $600 \mathrm{mg}$ (child $20 \mathrm{mg} / \mathrm{kg}$ ) [6].

Regimens for dental prophylaxis should always be given 30 to 60 minutes before the procedure. Oral amoxicillin remains the drug of choice. Ampicillin, Cefazolin, or ceftriaxone can be used, either 
intramuscularly or intravenously for penicillin-allergic patients [7].

Implications for orthodontic treatment in Infective endocarditis

1. Orthodontic procedures including impression making, placement of separators, banding, surgical exposure of impacted teeth can produce bacteraemia and therefore a drug regime shortly before the procedure should be given in order to kill causative micro organisms and reduce the risk.

2. As far as possible bonding should be preferred than banding.

3. Patient must be encouraged to use antimicrobial mouthwash to control plaque and maintain a high standard of oral hygiene.

4. It is desirable to prevent gingival bleeding before it occurs and it's best achieved by establishing and maintaining excellent oral hygiene.

5. Chronic irritation from orthodontic appliance may cause bleeding and special effort should be made to avoid any form of gingival or mucosal irritation.

6. Arch wire should be secured with elastomeric modules rather than wire ligatures and special care is required to avoid mucosal cuts when placing and removing the wire [8].

\subsection{Hypertension}

High blood pressure is a trait as opposed to a specific disease and represents a qualitative rather than a quantitative deviation from the norm. Any definition of hypertension is therefore arbitrary. High blood pressure is the major risk factor for cardiovascular disease and a major cause of renal failure and stroke. A blood pressure reading of $>160 / 95 \mathrm{~mm} \mathrm{Hg}$ is generally considered to represent hypertension for adults. A reading between 140/90 $\mathrm{mm} \mathrm{Hg}$ and 160/95 $\mathrm{mm} \mathrm{Hg}$ is borderline hypertension [9].

Elective dental treatment for uncontrolled hypertensive patients should be deferred until control is achieved. There is no contraindications, however to provide orthodontic care for well-controlled hypertensive patients. During treatment the orthodontist should be particularly vigilant for any deterioration in gingival health as Calciumchannel blocking drugs, especially nifedipine, have been associated with gingival overgrowth $[10,11,12]$ special efforts should be made to avoid any form of gingival irritation and the orthodontist should get the physicians appraisal before start of the treatment.

Gingival enlargement is not a true hyperplasia but involves proliferation of noncellular connective tissue elements of the gingival and the reaction occurs most prominently on the labial surfaces of the incisors. Though the Local plaque accumulation and inflammation are involved in the etiology of gingival overgrowth, and meticulous oral hygiene should be maintained by patients wearing the braces. Surgical resection of the enlarged tissues is effective in preventing recurrence [13]. Switching to an alternative antihypertensive drug addresses the problem successfully without relying on the patient's skill with the toothbrush. The Common drugs used in hypertensive patients are Diuretics (e.g., furosemide, hydrochlorothiazide, $\beta$-Adrenergic receptor blockers (e.g., propranolol, metoprolol) Nonselective $\beta$ - blockers (e.g., propranolol) ACE inhibitors (e.g., captopril) centrally acting $\alpha 2$-adrenergic receptor agonists (e.g., clonidine) Peripheral adrenergic neuron blockers (e.g., guanethidine).

\section{Implications for orthodontic treatment in} Hypertension

1. Minimizing stress is important.

2. It has been recommended that appointments should be less than one hour to minimize stress.

3. Maintaining periodontal health and good oral hygiene, educating the patient on this, and recommending specific oral hygiene aids and devices are important components of care for the patient with cardiovascular disease.

4. Calcium channel blockers can cause gingival hyperplasia in addition to the irritation caused by the fixed appliance. Depending on the condition refer the patient back to his physician or cardiologist, to prescribe an alternative therapy.

\subsection{Diabetes Mellitus}

Diabetes mellitus is one of the most common endocrine disorders [14,15]. It is a clinical syndrome characterised by persistently raised blood glucose levels (hyperglycaemia) resulting from deficiencies in insulin secretions, insulin action or both. The classical symptom of marked hyperglycaemia includes polyuria, polydyspia, weight loss and susceptibility to infections. Also, increased tendency for periodontal disease is often found in people with diabetes. The determination of the glucose concentration in a properly obtained venous sample is the only reliable criterion to establish the diagnosis of DM.

Diabetes affects individuals of all ages with a peak incidence in the 5th decade of life. Two major forms of this condition are type I, insulin dependent diabetes mellitus (IDDM) and Type II, non-insulin dependent diabetes mellitus (NIDDM).Due to rapid and severe onset of symptoms, type1 diabetes is often diagnosed at adolescence and was therefore termed "juvenile onset diabetes”. Type 1 diabetes is most common in childhood and adolescence and has a peak incidence at the age of 10 to 14 years $[16,17,18]$.

Type 2 diabetes, the cause is a combination of resistance to insulin action and an inadequate compensatory insulin secretor response in this type of diabetes ,the hyperglycaemia develops gradually and starts usually at the age of 40 or later [19]. Many patients have only moderately raised blood glucose levels. In the early stage of disease, classical symptoms of diabetes are usually not severe to alert the patient. Due to this slow rate of clinical onset, type 2 diabetes often remains undiagnosed.

Factors that may contribute to oral complication in diabetes include decreased polymorphonu clear (PMN) and leucocyte function and collagen metabolism. In addition, impaired neutrophil chemotaxis and macrophage functions add to impaired wound healing in diabetes patients [20,21]. The orthodontist should be aware of the significance of diabetes in relation to susceptibility to periodontal breakdown and hence patients with poorly controlled IDDM should not be treated. If the patient is diagnosed, DM during the orthodontic treatment the orthodontist should monitor the patient and the importance 
of diligently following the oral hygiene should be emphasised. Light force should be applied and vitality of the tooth should be checked in every visit. Patient with well controlled diabetes are no contraindication for orthodontic treatment.

Implications for orthodontic treatment in Diabetes mellitus

1. Orthodontic treatment should be avoided in patients with uncontrolled or poorly controlled diabetes. As they are susceptible to periodontal breakdown.

2. Periodontal condition should be monitored every visit and the patient should be with good oral hygiene and without local factors such as calculus as they are prone for gingival inflammation due to impaired neutrophil function.

3. Check for HbA1c or contact the patient's physician to verify the control of the disease.

4. Light forces should only be applied on the tooth vitality of the teeth involved should be checked on a regular basis.

5. Early appointment to be scheduled after breakfast.

6. There is no treatment preference with regard to fixed or removable appliances. It is important to stress the maintenance of good oral hygiene, especially when fixed appliances [22].

\subsection{Epilepsy}

Epilepsy means a tendency to have seizures and is a symptom of brain disease rather than a disease itself. The classification of epilepsy is achieved by considering the clinical events [23]. though the dental health should concern everyone; it is of particular importance to those who regularly take medication including many children and adults with epilepsy.

Gingival hypertrophy associated with anticonvulsant medication and past dental or facial trauma should be considered by the orthodontist when planning treatment and reviewed as part of patient informed consent. Some anti-epileptic drugs and other medications can cause dental problems. Symptoms such as bad breath, or gums being sore, swollen or red, may indicate a need to pay closer attention. It is well known that phenytoin causes gingival hyperplasia results in painful, swollen, red gums in a majority of patients. Studies have reported that the drug induces fibroblasts and osteoblasts, that there is an excessive deposition of extracellular matrix, and that normal tissue turnover and wound healing are altered [24].

Recent studies have report facial and body asymmetries affecting $41 \%$ of patients with partial seizures [25] If antiepileptic drug is been introduced in high doses at an early age, the position of the teeth and supporting bone structure, and the development of the child's skull, can all be affected to varying degrees. Therefore, it is very important to pay close attention to the development of teeth and to treat problems, which may include teeth growing out of alignment. The chances of gum tissue overgrowth, which makes biting and eating difficult and hence may sometimes require trimming of the gum line before placement of the brackets.

Medical help is required if any fragments have been swallowed or inhaled, while the jaw is clenched during a seizure .Falling can sometimes cause problems to soft and hard oral tissues. People who experience drop attacks (both tonic and atonic) are more likely to sustain dental injuries. Epilepsy and dental care both require regular monitoring and an orthodontist should assess the treatment protocol for such patient and should be a part of a comprehensive lifelong dental care.The hypertonicity of the oral musculature has caused the protrusion of the anterior teeth and the orthopaedic compression of the maxilla.

Anti-epileptic drugs related to oral findings include recurrent aphthous-like ulcerations, gingival bleeding, hypercementosis, root shortening, anomalous tooth development, delayed eruption, and cervical lymphadenopathy [26].

\section{Implications for orthodontic treatment in Epilepsy}

1. Appointments should be scheduled during a time of day when seizures are less likely to occur, if predictable, and stress and anxiety should be minimized.

2. Patients with epilepsy and a malocclusion should have a comprehensive orthodontic evaluation. Investigative procedure such as MRI should be taken prior to treatment as the metal components may distort the image.

3. Antiepileptic drugs can cause xerostomia, ulcerations, xerostomia, glossitis, and stomatitis hence the patients should be put on supplemental topical fluoride to prevent dental decay and monitored regularly for candidal infections.

4. The frequency of dental check-ups and prophylaxis appointments should be based on the patient's needs.

5. Consider use of mouth prop at the beginning of procedure. If a seizure occurs while a patient is in the dental chair. Firstly, clear all instruments away from the patient. Place the dental chair in a supported, supine position as near to the floor as possible.

\subsection{Pregnancy}

Among systemic factors, the role of hormonal changes during pregnancy is well established. While presence of fixed orthodontic appliances alone may not cause gingivitis, factors such as pregnancy and poor oral hygiene combined together could precipitate acute gingival inflammation that may progress to a periodontal condition in a patient receiving orthodontic therapy. There has been an increase in the number of adult patients who are receiving orthodontic treatment.

Orthodontic appliances could act as a potential plaque retentive source and aggravate inflammatory reactions that are seen during pregnancy. Most pregnant patients are generally healthy and need not be denied dental treatment solely because they are pregnant. Pregnancy gingivitis usually appears in the first trimester of pregnancy. This form of gingivitis results from increased level of progesterone and oestrogen causing an exaggerated gingival inflammatory reaction to local irritants. It is prudent to keep the pregnant patients periodontal system as free of disease as possible. Optimal oral health is very important for the pregnant patient and can be provided safely and effectively. Paying attention to the physiologic changes associated with pregnancy, practising careful radiation hygiene measures, prescribing medications based on drug safety categories and timing appointments and aggressive management of oral infection appropriately are 
important considerations. Given the possibility that periodontal disease may affect pregnancy outcomes, dentists need to play a proactive role in the maintenance of the oral health of pregnant women.

Patients have a heightened awareness of and sensitivity to taste, smell and environmental temperature. Unpleasant tastes and odours can cause severe nausea or even gagging and vomiting, Patient should be well hydrated, and the duration of chair treatment time should be as short as possible.

Coronal scaling, polishing and root planning may be performed at any time as required to maintain oral health. Oral radiography is safe for pregnant patients, provided protective measures such as high-speed film, a lead apron and a thyroid collor are used. No increase in congenital anomalies are intrauterine growth retardation has been reported for xray radiation exposure during pregnancy totalling less than 5 - $10 \mathrm{cGy}$, and a full mouth series of dental radiographs results in only $8 \times 10-4$ cGy. A bite wing and panoramic radiographic study generates about one-third the radition exposure associated with a full mouth series with E-speed film and a rectangular collimated beam the dental staff will practise the ALARA (as low as reasonably achievable) principle and that only radiographs necessary for diagnosis will be obtained [27].

Implications for orthodontic treatment in Pregnancy

1. Avoid X-rays or drug therapy, particularly in the first trimester

2. Extensive elective procedures, such as surgical exposure of impacted tooth should be postponed until after delivery.

3. Patients should be well hydrated, and the duration of chair treatment time should be as short as possible.

4. Maintaining a healthy oral environment can prevent potential problems

\subsection{Thyroid Disorders}

The thyroid gland and its hormones play an important role in the regulation of growth, development and metabolic functions of the body. Thyroid diseases include a group of condition that can affect the dental care. As part of a health care team, the dentist plays an important role in detecting thyroid abnormalities. Modifications of dental care must be considered when treating patients who have thyroid disease [28]. Thyroid hormones influence the growth and maturation of tissue, energy metabolism and turnover of both cells and nutrients.

The internal anatomy of the thyroid gland consists of follicles that contain mucinous colloid where the protein thyroglobulin is found. Thyroglobulin is the basic building block for the two main hormones produced by the thyroid; triiodothyronine or T3 and thyroxine or T4. In addition to thyroglobulin, iodine is needed for T3 and T4 synthesis Iodine is transported into the thyroid follicular cells and is combined with thyroglobulin to form the thyroid hormone precursor. A deficiency of either T4 or T3 can effect adversely the growth and development of the infant and will decrease metabolic function in the adult. An overproduction or excess availability of thyroid hormones can cause serious and life-threatening complications if not discovered and managed in time. Due to negative feedback mechanism regulating thyroid hormone secretion, the measurement of serum TSH, is the best test to determine thyroid function. People who have primary hypothyroidism will have increased TSH concentration because of the body's attempt to produce more thyroid hormone. Common oral findings in hypothyroidism include macroglossia, dysgeusia, delayed eruption, poor periodontal health and delayed wound healing [29]. Patient who are on a stable dosage of hormone replacement for a long time should have no problem withstanding routine and emergent orthodontic treatment.

Before treating a patient who has a history of thyroid disease, the dentist should obtain the correct diagnosis and etiology for the thyroid disorder, as well as past medical complications and medical therapy. In patients who have hypothyroidism are more susceptibility to cardiovascular disease and elevated LDL. Before treating such patients, consultation for their cardiovascular statuses should be taken [30] the main manifestation of hyperthyroidism in young patients is Graves' disease, while middle-aged men and women present most commonly with toxic nodular goiter.

Taking a careful history and conducting a thorough physical examination can indicate to the oral health care professional the level of thyroid hormone control of the patient. Patients who have hyperthyroidism are susceptible to cardiovascular disease from the ion tropic and chronotropic effect of the hormone, which can lead to atrial dysrhythmias [31,32].

Patients who have hyperthyroidism have increased levels of anxiety and stress. Surgery can trigger a thyrotoxic crisis. Medications such as epinephrine and other vasopressor amines should be given with caution in patients with treated hyperthyroidism and elective dental care should be deferred for patients who have hyperthyroidism and exhibit signs or symptoms of thyrotoxicosis. Patients with hypothyroidism require careful consideration due to the potential for excessive sedation [33,34,35]. Treatment procedures such as banding and bonding should have brief appointments and stress management are important for patients who have hyperthyroidism.

Implications for orthodontic treatment in Thyroid

1. Avoid severe stress situations, use of adrenaline and the spread of infectious foci [36].

2. Treatment should be discontinued if signs or symptoms of a thyrotoxic crisis develop and access to emergency medical services should be available.

3. After treatment it is important that patients continue taking their thyroid medication as prescribed.

4. One way the dental professional can protect the thyroid as it is extremely sensitive to radiation, and excessive radiation exposure is a known risk factor for various thyroid conditions. Thyroid collar is a must while taking patient X-rays [37].

\subsection{Hepatitis}

Hepatitis means inflammation of the liver, as well as referring to a group of infections caused by different viruses that affect the liver. The most common types are hepatitis A, hepatitis B, and hepatitis $C$, while hepatitis D and $\mathrm{E}$ are less common [38].

The nature of dental procedures routinely involves the risk of occupational exposure to blood, saliva, or other body fluid that might carry disease germs, which could 
infect dental personnel [39]. Orthodontic treatment often involves contact with blood and blood-contaminated saliva, Many individuals who are infected with variety of pathogens are asymptomatic and the ease of transmission of blood-borne diseases are through a single exposure incident which might result in infection and subsequent illness and, in some cases even death. Hepatitis B virus (HBV) transmission is through patient-to-patient due to improper or incomplete removal of blood strains from the instruments [40].

Therefore, the philosophy in any dental practice should be to assume that any patient, or any person working in the dental office, might carry pathogens and all phases of treatment in a clinic should minimize the risk of crossinfection. As Orthodontists deals with the younger population of patients and it is indicated that approximately $25 \%$ of new HIV infected patients are under 20 years of age [41]. Instruments used on these patients are carried in the instrument processing area cautiously so as to prevent cross contamination .One should wear heavy utility gloves and personal protective equipment during the decontamination procedure Also, at younger ages acute HBV infections are asymptomatic with less than $10 \%$ of them exhibiting jaundice, and the younger the person is at the time of infection, the higher the risk of developing chronic HBV infection [42].

Full-banded orthodontic appliances are commonly associated with an increased bacterial accumulation and can compromise both the self-cleansing component of the dentition and the ability of the patient to effectively remove plaque. Thus, hyperplastic marginal gingivitis is an almost inevitable result of poor plaque control in patients undergoing orthodontic treatment [43,44]. In addition, among all orthodontic procedures, banding and debanding are considered to cause greatest damage to the gingival margins [45] As a result, gingival inflammation and bleeding are usually encountered during the de bonding procedure [46].

Implications for orthodontic treatment in Hepatitis

1. Protecting patients from cross-contamination and preventing exposure of office staff to infectious diseases should be the first priority.

2. A one-tier cross infection control policy should be adopted routinely.

3. Comprehensive and current medical and dental histories should be taken

4. Appropriate laboratory investigations are essential and if any abnormal levels are discovered, consultation with a hematologist or hepatologist is suggested before beginning orthodontic treatment.

\section{Conclusion}

Many patients seeking dental care have significant medical conditions that alter both the course of their oral disease and the therapy provided. Treatment of medically compromised orthodontic patients should be directed towards the prevention of oral complication that could be life threatening and hence special precautions are usually required. Decreased resistance to infection is a common complicating factor in medically compromised patients therefore any mucosal irritation should be monitored and regular periodontal evaluation should be done. With appropriate management, successful orthodontic treatment can be done with minimal physical damage and maximum treatment outcome.

\section{References}

[1] Durack DT, Lukes AS, Bright DK. New criteria for diagnosis of infective endocarditis: utilization of specific echocardiographic findings: Duke Endocarditis Service. Am J Med. 96: 200-209, 1994.

[2] Keys TF. Infetive endocarditis: Preventive, Diagnosis and treatment, referrals. Cleve clinical j Med 2000; 67: 353-360.

[3] Cabell CH, Abrutyn E, Karchmer AW. Cardiology patient page. Bacterial endocarditis: the disease, treatment, and prevention. Circulation 2003; 107: e 185-7.

[4] Heart disease diagnosis and therapy. Contemporary Cardiology, 2005, 415-426.

[5] Dajani AS, Taubert KA, Wilson W, et al. Prevention of bacterial endocarditis. Recommendations by the American Heart Association. JAMA. 1997; 277: 1794-1801.

[6] British dental association advisory services. Infection control in dentistry. American Journal of Infection Control 1996; 24: 313-42.

[7] Kim A, Keys T. Infective endocarditis prophylaxis before dental procedures: New guidelines spark controversy. Cleve Clin J Med. 2008; 75: 89-92.

[8] Donald burden, Brain Mullally, Jonathan sandler, Orthodontic treatment of patients with medical disorders, European journal of orthod 2001: 23; 363-372.

[9] Kilmaratin C, Munroe CO. Cardiovascular disease and the dental patients. Can Dent Assoc J 1986; 52: 513-518.

[10] Ciancio SG. Medications impact on oral health. J Am Dent Assoc 2004; 135: 1440-1448.

[11] Yagiela JA. Agents affecting salivation. In, Ciancio SG, ADA Guide to Dental Therapeutics, ed. 4. Chicago, ADA Publishing Co., 2006.

[12] Miranda J, Brunet L, et al, Prevalence and risk of gingival enlargement in patients treated with nifedipine. J Periodontol. 2001; 72: 605-11.

[13] Yagiela JA, Haymore TL. Management of the hypertensive dental patient. J Calif Dent Assoc 2007; 35: 51-59.

[14] Finney LS, Finney MO, Gonzalez-Campoy JM. What the mouth has to say about diabetes. Careful examinations can avert serious complications. Postgrad Med. 1997; 102: 117-26.

[15] Loe H. Periodontal disease: The sixth complication of diabetes mellitus. Diabetes Care 1993: 16; 329-334.

[16] Brownlee M, Cerami A. The biochemistry of the complications of diabetes. Annu Rev Biochem. 1981; 50: 385-432.

[17] Meyle J, Gonzáles JR. Influences of systemic diseases on periodontitis in children and adolescents. Periodontol 2000. 2001; 26: 92-112.

[18] Dokheel TM. An epidemic of childhood diabetes in the United States? Evidence from Allegheny County, Pennsylvania. Diabetes Care. 1993; 16: 1606-11.

[19] Bensch L, Braem M, Willems G. Orthodontic Considerations in the Diabetic Patient Seminars in Orthod 10: 252-258.

[20] Amos AF, McCarty DJ, Zimmet P. The rising global burden of diabetes and its complications: estimates and projections to the year 2010 Diabet Med. 1997; 14 Suppl 5: S 1-85.

[21] Saadoun AP. Diabetes and Periodontal Disease: a review and update. J West Soc Periodontol Periodontal Abstr. 1980; 28 (4): 116-39.

[22] Luc Bensch, Marc Braem and Guy Willems; Orthodontic Considerations in the Diabetic Patient; Semin Orthod 2004; 10: 252-258.

[23] Burden D, Mullally B, Sandler J. Orthodontic treatment of patients with medical disorders. Eur J Orthod. 2001 23: 363-72.

[24] Cornacchio AL, Burneo JG, Aragon CE. The effects of antiepileptic drugs on oral health. J Can Dent Assoc. 2011; 77: b 140.

[25] Fong, G. C, Mak, Y. F.; Schwartz, B. E.; Walsh, G. O. Body part asymmetry in partial seizure. Seizure, 2003: 12; 606-612.

[26] Johnston, S. C.; Barnard, K. M. \& Harrison, V. E. Recognizing and caring for the medically compromised child: 4 Children with other chronic medical conditions. Dent Update, 1999; 26: 21-26.

[27] Giglio JA, Lanni SM, Laskin DM, Giglio NW. Oral health care for the pregnant patient. J Can Dent Assoc 2009; 75: 43-48.

[28] Pinto A, Glick M. Management of patients with thyroid disease: oral health considerations. J Am Dent Assoc. 2002; 133: 849-58. 
[29] Young ER. The thyroid gland and the dental practitioner. J Can Dent Assoc 1989; 55: 903-907

[30] Muzyka BC. Atrial fibrillation and its relationship to dental care. J Am Dent Assoc 1999; 130: 1080-1085.

[31] Woeber KA. Thyrotoxicosis and the heart. N Engl J Med. 1992; 327: 94-8.

[32] Vanderpump MP, Tunbridge WM, French JM et al. The incidence of thyroid disorders in the community: a twenty-year follow-up of the Whickham Survey. Clin Endocrinol 1995; 43: 55-68.

[33] Burket's Oral Medicine Diagnosis \& Treatment, Tenth Edition, 2002, pg 341-571.

[34] Little: Dental Management of the Medically Compromised Patient, 2007, 7th edition

[35] Dental Secrets SE. Stephen TS. II nd edition-Pg 37-64.

[36] Carlos Fabue L, Jiménez Soriano Y, Sarrión Pérez MG. Dental management of patients with endocrine disorders. J Clin Exp Dent. 2010; 2 (4): e 196-203.

[37] Shalu Chandna, Manish Bathla, Oral manifestations of thyroid disorders and its management. Indian J Endocrinol Metab. 2011 July; 15: 113-116.

[38] Palenik CJ. Alphabet soup: The various hepatitis viruses. Contemporary Dental Assisting. 2007: Jan; 22-25.
[39] Samaranayake LP. Essential Microbiology for Dentistry. 2nd ed. Edinburgh: Churchill Livingstone; 1998: 263-320.

[40] Redd JT, Baumbach J, Kohn W, et al. Patient-to-patient transmission of hepatitis B virus associated with oral surgery. J Infect Dis. 2007; 195: 1311-1314.

[41] McCarthy GM, Mamandras AH, MacDonald JK. Infection control in the orthodontic office in Canada. Am J Orthod Dentofacial Orthop 1997; 112: 275-281.

[42] McMahon BJ, Alward WL, Hall DB et al. Acute hepatitis B virus: relation of age to the clinical expression of disease and subsequent development of the carrier state. J Infect Dis. 1985; 151: 599-603.

[43] Zachrisson S, Zachrisson BU. Gingival condition associated with orthodontic treatment. Angle Orthod. 1972; 42: 26-34.

[44] Heintze SD, Jost-Brinkmann PG, Finke C, Miethke R. Oral Health for the Orthodontic Patient. Chicago, Ill: Quintessence Publishing Co Inc; 1999: 1-23.

[45] Degling TE. Orthodontics, bacteremia, and the heart damaged patient. Angle Orthod. 1972; 42: 399-401.

[46] Erverdi N, Biren S, Kadir T, Acar A. Investigation of bacteremia following orthodontic debanding. Angle Orthod. 2000; 70: 11-14. 